\title{
Clinical features of lenvatinib treatment in elderly patients with advanced thyroid cancer
}

\author{
KOICHI SUYAMA ${ }^{1}$, SAORI FUJIWARA ${ }^{2}$, TAKASHI TAKESHITA ${ }^{2}$, AIKO SUETA $^{2}$, TOUKO INAO ${ }^{2}$, \\ MUTSUKO YAMAMOTO-IBUSUKI ${ }^{2}$, YUTAKA YAMAMOTO ${ }^{2}$ and HIROTAKA IWASE ${ }^{1,2}$ \\ ${ }^{1}$ Kumamoto University Hospital Cancer Center, Graduate School of Medical Sciences; \\ ${ }^{2}$ Department of Breast and Endocrine Surgery, Kumamoto University, Kumamoto 860-8556, Japan
}

Received October 13, 2016; Accepted March 29, 2017

DOI: $10.3892 / \mathrm{mco} .2017 .1265$

\begin{abstract}
Until recently, there had not been an effective systemic chemotherapy for advanced differentiated thyroid carcinoma (DTC); lenvatinib, a multi-tyrosine kinase inhibitor, has been proven effective for DTC, but has also been revealed to have adverse side effects including hypertension, hand-foot syndrome (HFS) and diarrhea. There have been few clinical studies focused on the characteristics, safety concerns or precautions for lenvatinib treatment in elderly patients. The present study administered lenvatinib to 18 patients with DTC in Kumamoto University Hospital (Kumamoto, Japan), with 9 patients in both the younger group ( $<75$ years old) and elderly group ( $\geq 75$ years old). The median maximum systolic blood pressure (sBP) was significantly different between the two groups $(158 \mathrm{mmHg}$ in the younger group vs. $173 \mathrm{mmHg}$ in the elderly group; $\mathrm{P}=0.042)$. There were no significant differences in median maximum diastolic blood pressure (94 vs. $95 \mathrm{mmHg}$; $\mathrm{P}=1.00$ ), median degree of $\mathrm{sBP}$ elevation (43 vs. $55 \mathrm{mmHg}$; $\mathrm{P}=0.199)$ or median days until hypertension diagnosis ( 2.11 vs. 2.33 days; $\mathrm{P}=0.436$ ). There were also no significant differences in other toxicities (HFS, proteinuria or diarrhea). In conclusion, lenvatinib should be introduced carefully to elderly patients with DTC, as they tend to present with hypertension during treatment. However, there were no differences in other toxicities between the younger and elderly groups; lenvatinib was fully tolerated in patients with DTC $>75$ years old.
\end{abstract}

\section{Introduction}

Differentiated thyroid carcinoma (DTC) typically has a good prognosis; however, effective systemic chemotherapy for advanced DTC had not existed until recently, when lenvatinib, a multi-tyrosine kinase inhibitor was proven an effective treat-

Correspondence to: Dr Koichi Suyama, Kumamoto University Hospital Cancer Center, Graduate School of Medical Sciences, Kumamoto University, 1-1-1 Honjo, Kumamoto 860-8556, Japan E-mail:kou_susan@yahoo.co.jp

Key words: thyroid cancer, targeted therapy, lenvatinib, hypertension, elderly ment option (1). Subsequently, lenvatinib has been introduced into clinical practice for advanced DTC. Unfortunately, there are little safety data for lenvatinib, particularly regarding elderly patients.

Numerous adverse effects of lenvatinib have been reported, including hypertension, hand-foot syndrome (HFS) diarrhea and thrombocytopenia. In the SELECT trial, $70 \%$ of patients receiving lenvatinib presented with hypertension (1); similar results have also been demonstrated in other clinical trials (2-4). Zhu et al (5) analyzed the safety and efficacy profiles of lenvatinib in patients with cancer in a systematic review and meta-analysis; in an analysis of 978 patients treated with lenvatinib, the most frequently observed adverse events of grade 3 or higher were thrombocytopenia (25.4\%), hypertension (17.7\%) and peripheral edema (15.5\%). Additionally, incidences of all-grade and high-grade hypertension were significantly increased (5).

Numerous problems have been reported regarding the use of chemotherapy in elderly patients (6-8). Elderly patients more frequently suffer from the adverse side effects of anticancer chemotherapy than younger patients. There are many clinical reports on the safety and efficacy of lenvatinib $(1,5)$, but none specifically study its safety profile in elderly patients. In clinical practice, lenvatinib is commonly used to treat elderly patients, and there is significant experience in administrating lenvatinib to elderly patients in Kumamoto University Hospital. The present study examined the side effects of lenvatinib in 18 patients grouped by age (younger, $<75$ years and elderly, $\geq 75$ years) to analyze differences in the adverse events associated with lenvatinib treatment in elderly patients.

\section{Patients and methods}

Patients. This retrospective, observational, cross-sectional study was designed to evaluate the safety of lenvatinib for elderly patients. A total of 18 consecutive patients with histopathologically-proven DTC treated with lenvatinib at Kumamoto University Hospital between July 2015 and July 2016 were enrolled in the study. Written informed consent was obtained from all patients prior to enrollment. Information regarding adverse events was obtained from medical charts and compared between younger ( $<75$ years) and elderly ( $\geq 75$ years) patients. When collecting blood pressure (BP) data, the hospital 
Table I. Patient characteristics.

\begin{tabular}{lc}
\hline Characteristic & No. of patients $(\%)$ \\
\hline Total & $18(100)$ \\
Age, median (range), years & $74(59-87)$ \\
$\geq 75$ & $9(50)$ \\
Sex & \\
Female & $9(50)$ \\
Male & $9(50)$ \\
ECOG performance status & \\
0 & $12(67)$ \\
1 & $5(28)$ \\
2 & $1(5)$ \\
Histology subtype & $18(100)$ \\
Papillary & \\
Prior treatment & $13(72)$ \\
None & $5(28)$ \\
Sorafenib & \\
History of hypertension & $11(61)$ \\
Yes & $7(39)$ \\
No & \\
Using antihypertensive drugs & $10(56)$ \\
Yes & \\
No & \\
\hline
\end{tabular}

ECOG, Eastern Cooperative Oncology Group.

$\mathrm{BP}$ was used rather than the home BP. Adverse events were evaluated using the Common Terminology Criteria for Adverse Events version 4.0 (9). The study protocol has been approved by the institutional review board of Kumamoto University.

Statistical analysis. The Mann-Whitney U test was used to compare the degree of each adverse event (such as BP), and $\chi^{2}$ tests were used to compare the proportions of variables between groups. All statistical analyses were conducted using SAS JMP Pro v12.1.0 (SAS Institute Inc., Cary, NC, USA). $\mathrm{P}<0.05$ was considered to indicate a statistically significant difference.

\section{Results}

Clinical characteristics of the 18 enrolled patients are presented in Table I. The proportion of younger and elderly patients and of males and females were the same in the overall cohort and groups. All patients had histologically-confirmed papillary thyroid carcinoma as their primary diagnosis and were administered $24 \mathrm{mg}$ per day lenvatinib, with the exception of 5 patients whose tumors showed vessel invasion (Table II).

The median maximum systolic BP (sBP) was significantly different between the younger patient and elderly patient groups (158 vs. 173 mmHg; P=0.042; Fig. 1A). There were no significant differences between the younger and elderly patients in median maximum diastolic $\mathrm{BP}(\mathrm{dBP} ; 94$ vs. $95 \mathrm{mmHg}$; $\mathrm{P}=1.00$;
Table II. Starting dose of lenvatinib.

\begin{tabular}{lcc}
\hline Starting dose, mg & No. of patients (\%) & Reason \\
\hline 24 & $13(72)$ & Standard dose \\
20 & $1(5)$ & Vessel invasion \\
14 & $3(18)$ & Vessel invasion \\
10 & $1(5)$ & Vessel invasion \\
\hline
\end{tabular}

A

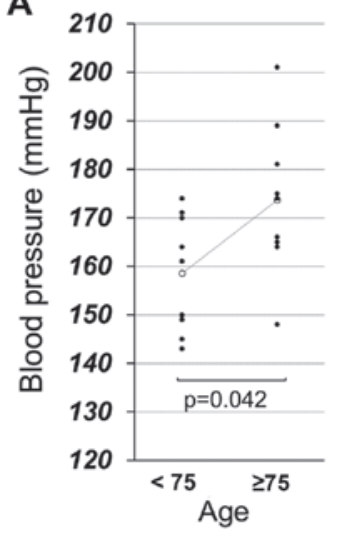

C

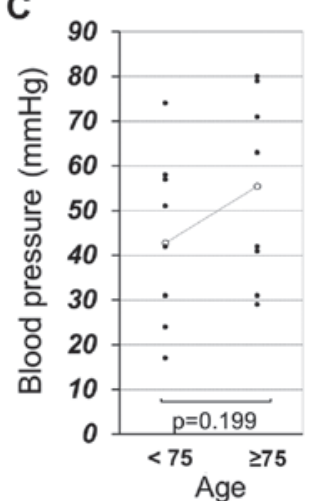

B

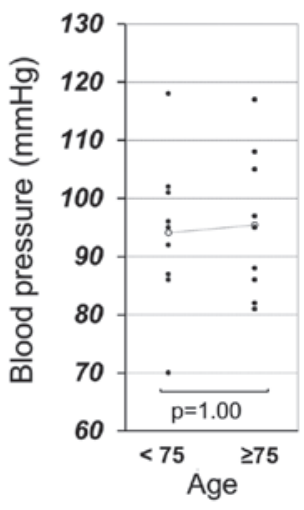

D

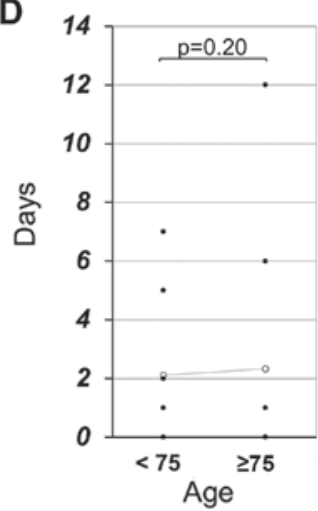

Figure 1. Comparison of the effects of lenvatinib on hypertension in patients with differentiated thyroid carcinoma $<75$ and $\geq 75$ years old. (A) Patients $\geq 75$ years old had significantly higher systolic blood pressure than patients $<75$ years old. There were no significant differences between younger $(<75)$ and elderly $(\geq 75)$ patients in (B) maximum diastolic blood pressure, (C) systolic blood pressure elevation or (D) median number of days before hypertension diagnosis.

Fig. 1B), median degree of sBP elevation (43 vs. $55 \mathrm{mmHg}$; $\mathrm{P}=0.199$; Fig. 1C) or median days until a clinical hypertension diagnosis (2.11 vs. 2.33 days; $\mathrm{P}=0.436$; Fig. 1D). There was also no significant difference in the baseline sBP between elderly and young patients. Furthermore, the frequency of other adverse events (grade $\geq 1$ HFS, proteinuria and diarrhea) exhibited no significant differences between groups (Table III).

Between the two groups, there were no significant differences in the proportion of patients with hypertension history and using antihypertensive drugs.

\section{Discussion}

The present study performed a retrospective analysis of 18 consecutive lenvatinib-treated advanced DTC patients from 
Table III. Effects of lenvatinib on HFS, proteinuria and diarrhea.

\section{A, HFS}

\begin{tabular}{lcc}
\hline Age & HFS (yes/no) & P-value \\
\hline$<75$ & $3 / 6$ & 0.08 \\
$\geq 75$ & $5 / 4$ & \\
\hline
\end{tabular}

\section{B, Proteinuria}

\begin{tabular}{lcc}
\hline Age & Proteinuria (yes/no) & P-value \\
\hline$<75$ & $6 / 3$ & 0.08 \\
$\geq 75$ & $2 / 7$ & \\
\hline
\end{tabular}

\section{C, Diarrhea}

\begin{tabular}{ccc}
\hline Age & Diarrhea (yes/no) & P-value \\
\hline$<75$ & $0 / 9$ & 1.0 \\
$\geq 75$ & $1 / 8$ &
\end{tabular}

Adverse events evaluated according to Common Terminology Criteria for Adverse Events version 4. HFS, hand-foot syndrome; yes, grade 1 or more; no, none.

Kumamoto University Hospital. The analysis was focused on comparing the degree of adverse events associated with lenvatinib treatment between elderly and younger patient groups. Among several adverse events, hypertension was focused upon, as it has previously been demonstrated to be a major clinical concern for elderly patients receiving chemotherapy (6-8). The results revealed that $\mathrm{sBP}$ was significantly elevated in elderly patients compared with younger patients, but the change from baseline to maximum sBP was not significant. As presented in Fig. 1C, the elderly patients had at least the trend to have more elevated sBP than young patients following lenvatinib therapy. This trend may be one of the reasons for elevated sBP in elderly patients. Conversely, there were no indications of increases in other lenvatinib-specific toxicities such as HFS, proteinuria, diarrhea, fatigue and thrombocytopenia among elderly patients.

Elderly patients suffer more chemotherapy-induced toxicities than younger patients with cancer (6-8). In clinical trials the majority of patients are relatively young and in good condition; thus, there is often a lack of information from these studies regarding efficacy and safety for elderly patients. Among studies investigating the efficacy of lenvatinib for thyroid carcinoma, to the best of our knowledge there have been no studies analyzing the degree of lenvatinib-specific adverse effects in elderly patients. The present results revealed a trend of increased maximum sBP in elderly patients treated with lenvatinib compared with younger patients; however, the frequency of other toxicities did not increase, indicating that lenvatinib is relatively safe for elderly patients.

In general, elderly patients tend to have increased sBP and decreased $\mathrm{dBP}$, caused by decreased elasticity and extensibility of large arteries occurring due to the extension of arteriosclerosis associated with increasing age. Although the precise mechanisms of BP elevation following lenvatinib treatment are unclear, one possible explanation may be lenvatinib-induced vascular endothelial cell injury (10). The underlying mechanism of the high sBP in elderly patients receiving lenvatinib may involve effects on the vascular endothelial cells that surround arteriosclerotic vessels. There is no evidence that demonstrates that elderly patients tend to have HFS, diarrhea and other toxicities, which might explain the lack of increased frequencies of these adverse events in patients over 75 in the present study.

There were several limitations to the current study. First, this was a retrospective observational study, not a case-controlled study; therefore, the study design cannot avoid confounding and selection biases. Second, the number of patients was small, the results are not able to be extrapolated without subsequent studies with larger patient cohorts. Thus, we cannot regard this study as high quality.

In conclusion, a trend of hypertension in elderly patients receiving lenvatinib but not in younger patients was observed, suggesting that lenvatinib should be introduced carefully to elderly patients. However, lenvatinib-induced hypertension may easily be controlled using anti-hypertensive drugs or adjusting the dose of lenvatinib. Overall, lenvatinib was tolerable, even in elderly patients $>75$.

\section{References}

1. Schlumberger M, Tahara M, Wirth LJ, Robinson B, Brose MS, Elisei R, Habra MA, Newbold K, Shah MH, Hoff AO, et al: Lenvatinib versus placebo in radioiodine-refractory thyroid cancer. N Engl J Med 372: 621-630, 2015.

2. Boss DS, Glen H, Beijnen JH, Keesen M, Morrison R, Tait B, Copalu W, Mazur A, Wanders J, O'Brien JP, et al: A phase I study of E7080, a multitargeted tyrosine kinase inhibitor, in patients with advanced solid tumours. Br J Cancer 106: 1598-1604, 2012.

3. Nakamichi S, Nokihara H, Yamamoto N, Yamada Y, Honda K, Tamura Y, Wakui H, Sasaki T, Yusa W, Fujino K and Tamura T: A phase 1 study of lenvatinib, multiple receptor tyrosine kinase inhibitor, in Japanese patients with advanced solid tumors. Cancer Chemother Pharmacol 76: 1153-1161, 2015.

4. Cabanillas ME, Schlumberger M, Jarzab B, Martins RG, Pacini F, Robinson B, McCaffrey JC, Shah MH, Bodenner DL, Topliss D, et al: A phase 2 trial of lenvatinib (E7080) in advanced, progressive, radioiodine-refractory, differentiated thyroid cancer: a clinical outcomes and biomarker assessment. Cancer 121: 27492756, 2015.

5. Zhu C, Ma X, Hu Y, Guo L, Chen B, Shen K and Xiao Y: Safety and efficacy profile of lenvatinib in cancer therapy: a systematic review and meta-analysis. Oncotarget 7: 44545-44557, 2016.

6. Chrischilles EA, Pendergast JF, Kahn KL, Wallace RB, Moga DC, Harrington DP, Kiefe CI, Weeks JC, West DW, Zafar SY and Fletcher RH: Adverse events among the elderly receiving chemotherapy for advanced non-small-cell lung cancer. J Clin Oncol 28: 620-627, 2010.

7. Townsley CA, Selby R and Siu LL: Systematic review of barriers to the recruitment of older patients with cancer onto clinical trials. J Clin Oncol 23: 3112-3124, 2005.

8. Clough-Gorr KM, Stuck AE, Thwin SS and Silliman RA: Older breast cancer survivors: geriatric assessment domains are associated with poor tolerance of treatment adverse effects and predict mortality over 7 years of follow-up. J Clin Oncol 28: 380-386, 2010.

9. US Department of Health and Human Services: National Cancer Institute Common Terminology Criteria for Adverse Events v4.0. https://evs.nci.nih.gov/ftpl/CTCAE/Archive/CTCAE_4.02 _2009-09-15_QuickRef erence_5x7_Locked.pdf. Accessed September 1, 2016.

10. Keizer RJ, Gupta A, Mac Gillavry MR, Jansen M, Wanders J, Beijnen JH, Schellens JH, Karlsson MO and Huitema AD: A model of hypertension and proteinuria in cancer patients treated with the anti-angiogenic drug E7080. J Pharmacokinet Pharmacodyn 37: 347-363, 2010. 